\title{
Condiciones óptimas para el tratamiento, con vapor, del hormigón
}

O. P. MTSCHEDLOW-PETROSSIAN, P. A. MELIITSCHENIKO, 1. 8. DMITRIIEW. V. L. W. SAWORIN

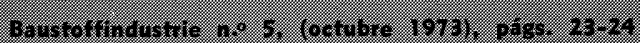

El fraguado acelerado se obtiene la mayor parte de las veces en hormigones de cemento mediante elevadas temperaturas y con una humedad relativa del aire del $100 \%$.

Si se aplica el tratamiento hidrotérmico, sin tener en cuenta los procesos físicos y físicoquímicos que se desarrollan durante el fraguado, hay que contar con un consumo elevado de cemento y un largo tratamiento térmico. Además, las consecuencias son: reducidas resistencias mecánicas y una insuficiente resistencia a la helada.

Si en el tratamiento hidrotérmico se excluyen las condiciones tecnológicas, las de la termodinámica, la cinética del proceso de fraguado [precalentamiento, calentamiento eléctrico, tratamiento termodinámico (1), (5), (6)] y se efectúa la vaporización bajo presión atmosférica, deben tenerse en cuenta los procesos físicos provocados por el calor que acompañan al fraguado acelerado (3). Esto es especialmente necesario porque la composición de fases de las nuevas formaciones, en el caso de hormigones tratados hidrotérmicamente, no se diferencia de las de los hormigones fraguados normalmente.

Casi siempre, el tratamiento hidrotérmico del hormigón aumenta la porosidad (1), y resulta peor la unión de adherencia entre el cemento y el árido (4). Para poder captar mejor la dependencia de las propiedades y de la estructura del hormigón respecto del régimen de calor y humedad, en la cátedra de elementos de construcción del Instituto S. M. Kirow se construyó una cámara de vapor. En ella se regulan, según un programa previamente fijado, la temperatura y la humedad y es posible examinar los procesos que se desarrollan durante el tratamiento de hidratación. Para garantizar un régimen exterior uniforme de temperatura-humedad, se introduce en la cámara una mezcla de vapor-aire bajo presión (2).

Las probetas de hormigón que hay que analizar $(100 \mathrm{~mm} \times 100 \mathrm{~mm} \times 100 \mathrm{~mm})$ se fabricaron en una mesa vibradora estándar y con moldes de acero. Se prepararon, cada vez, 12 probetas que forman un complejo para investigación.

Composición del hormigón:

\begin{tabular}{|c|c|}
\hline Cemento ....... & $430 \mathrm{~kg} / \mathrm{m}^{3}$ \\
\hline Gravilla ........ & $1.350 \mathrm{~kg} / \mathrm{m}^{3}$ \\
\hline Arena ... & $550 \mathrm{~kg} / \mathrm{m}^{3}$ \\
\hline Agua & $165 \mathrm{~kg} / \mathrm{m}^{3}$ \\
\hline
\end{tabular}

Al mismo tiempo se prepararon simultáneamente 9 probetas de pasta de cemento $(70 \mathrm{~mm} \times$ $\times 70 \mathrm{~mm} \times 70 \mathrm{~mm}$ ) formando otro complejo. 
El aluminato contenido en el cemento "Alit" empleado alcanzó un máximo del $6 \%$; la superficie específica según Towarow fue $s=2.900 \mathrm{~cm}^{2} / \mathrm{g}$.

En diferentes puntos de los cubos de hormigón se insertaron termómetros de resistencia, con el fin de poder medir la temperatura durante el tratamiento.

Para el fraguado acelerado del hormigón se estableció el siguiente plan de temperaturatiempo: 2 horas de precalentamiento; 3 horas de calentamiento hasta $80^{\circ} \mathrm{C}$; 3 horas de tratamiento isotérmico a temperatura máxima, y 3 horas de enfriamiento (tabla I).

\section{TABLA I}

Humedad relativa de la mezcla de vapor-aire en la cámara (\%)

\begin{tabular}{ccccc}
\hline $\begin{array}{c}\text { Régimen de } \\
\text { tratamiento }\end{array}$ & $\begin{array}{c}\text { Tiempo de } \\
\text { precalentamiento }\end{array}$ & Calentamiento & Tratamiento isotérmico & Enfriamiento \\
\hline 1 & 60 & 100 & 100 & 65 \\
2 & 60 & 30 & 30 & 30 \\
3 & 60 & 30 & 100 & 100 \\
4 & 60 & 100 & 30 & 100 \\
5 & 60 & 100 & 100 & 30 \\
6 & 60 & regulable & 100 & 100 \\
7 & Fraguado normal, $\dagger=18^{\circ} C^{*} w=98 \%$ & &
\end{tabular}

Durante el tratamiento hidrotérmico se anotaron continuamente las temperaturas del medio ambiente y de las muestras en diferentes secciones, la humedad relativa del medio así como el peso del cubo de hormigón.

Tras el tratamiento hidrotérmico fueron examinadas las probetas en relación con su resistencia a la compresión y la estabilidad en caso de carga dinámica. Además se determinaron la velocidad, carácter y orden de la absorción de agua así como la eventual deficiencia de hormigón. Para poder determinar el grado de hidratación así como el carácter y el volumen de la porosidad, se prepararon, de las probetas, placas delgadas.

\section{Análisis de los resultados}

En la tabla II se encuentran agrupados los valores de las resistencias, inmediatamente después del tratamiento y al cabo de 28 días.

TABLA II

Influencia del régimen de tratameinto en la resistencia del hormigón

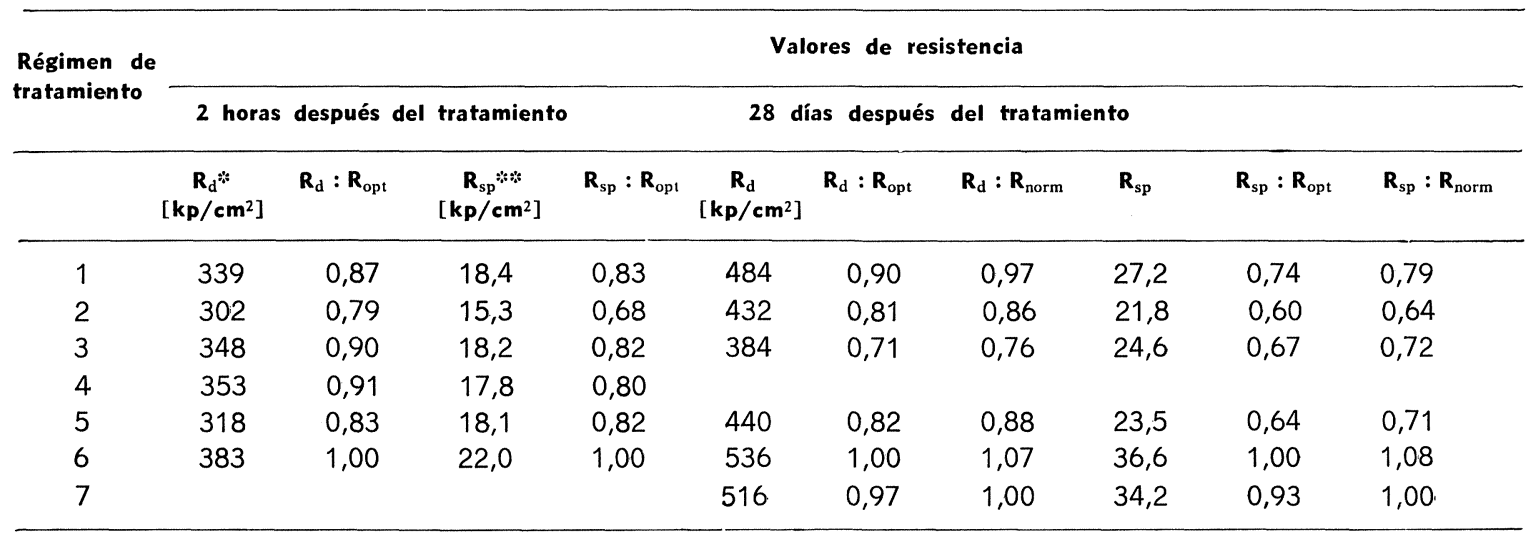

* Resistencia del hormigón a la compresión.

** Resistencia a la tracción por hendidura sobre la tabla estriada. 
En la figura 1 está representado el peso de la probeta que cambia durante el tratamiento; en la figura 2, la velocidad de calentamiento en el centro del cubo, dependiente del rágimen aplicado; y en la figura 3, la influencia de la humedad sobre la resistencia.

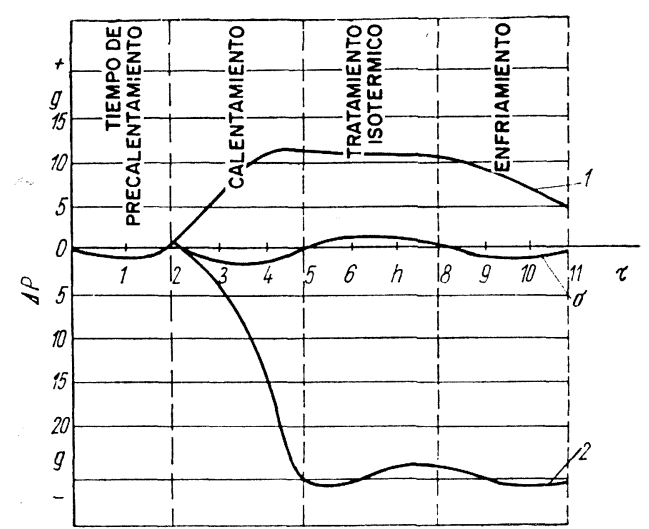

Fig. 1-Modificación del peso de la muestra de hormigón durante el tratamiento térmico.

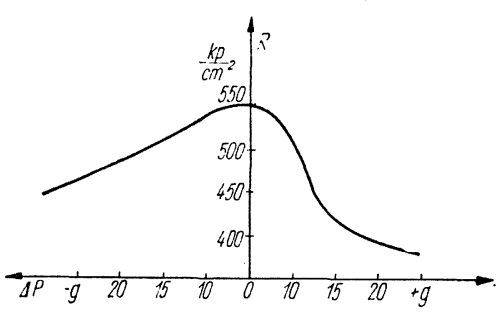

fig. 2.-Dependencia entre la resistencia del hormigón y la humedad inicial.

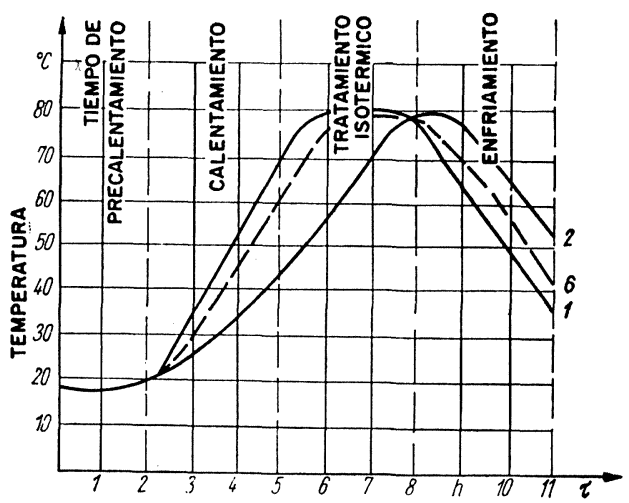

Fig. 3.-Desarrollo del calentamiento en el centro de la muestra.
El análisis de las tablas y diagramas demuestra que tanto los parámetros de los procesos de tratamiento térmico como también los valores distintivos del material obtenidos dependen de la humedad relativa del medio ambiente. Si la temperatura en la superficie de la probeta a tratar se encuentra durante el aumento de temperatura por debajo del punto de rocío del vapor de agua saturado, se llega a la condensación de vapor en la superficie. Debido a los cambios caloríficos, el producto condensado tiende a penetrar hacia el interior de la probeta; la presión que se origina contribuye a incrementar la resistencia del hormigón.

La humedad así condensada aumenta localmente el valor $a / c$ y aumenta, por tanto, la porosidad en la superficie de las probetas de hormigón.

Si el medio ambiente presenta un contenido reducido de humedad, el hormigón cede una parte considerable de la humedad propia, que se mueve en dirección a la superficie. Defectos o faltas del hormigón, originados durante el aumento de temperatura o que aparecen durante el tratamiento isotérmico, pueden compensarse por una marcha conveniente del enfriamiento. Al mismo tiempo la pérdida de humedad durante el tiempo de enfriamiento empeora de manera irreversible las propiedades del hormigón. La cantidad de humedad que se desplaza depende del desnivel de temperatura existente entre la mezcla vapor-aire y el hormigón, así como de la humedad relativa del medio ambiente. La humedad que se mueve en el hormigón relaja el material y produce una porosidad orientada.

Los procesos del intercambio exterior de calor y material se concentran sobre la superficie de la probeta, puesto que aquí se presentan las mayores alteraciones de su estructura, como lo han demostrado las investigaciones sobre la distribución de la porosidad en toda la sección de la probeta. La porosidad de la superficie es aproximadamente de 2 a 5 veces mayor durante el tratamiento térmico que en las zonas interiores. El intensificado intercambio de material produjo una notable alteración en la estructura y relajó la zona de contacto entre el árido y la pasta de cemento, lo que se confirmó mediante la "defectoscopia lumínica". 
Una consecuencia de la destrucción del material es su mayor absorción de agua (tabla III) y la estabilidad de 15 a 20 veces menor contra los esfuerzos dinámicos en régimen de saturación de agua. De este modo es necesario, para alcanzar una óptima estructura homogénea, reducir a un mínimo los procesos destructivos y mantener tan reducido como sea posible el intercambio externo e interior de material durante el tratamiento térmico. Además debe mantenerse a su nivel inicial el contenido de humedad del hormigón. Así se obtiene también con el tratamiento térmico un material de óptimos valores característicos físicomecánicos (resistencia, absorción de agua, resistencia a la helada, resistencia contra efectos dinámicos). Así se reduce la porosidad total, y resulta más uniforme la distribución de los poros. La investigación de las placas pulidas demostró tanto una buena unión entre la pasta del cemento y el árido, como una hidratación regular del cemento.

\section{B I B L I O G R A F I A}

(1) Berkowitsch, T. M., u. a.: Hydratationsprozesse bei der beschleunigten Erhärtung von Zement (russ). Arbeiten RILEM, Moskau 1962.

(2) Wosnessenski, A. A.: Bedampfung von Stahlbetonerzeugnissen mit intensiver Zirkulation des Erhitzungsmediums (russ.) Beton i Shelesobeton (1967), H. 12.

(3) Marjamow, N. B.: Warmbehandlung von Erzeugnissen in Betrieben für Stahlbetonfertigteile (russ.). Stroiisdat, 1970.

(4) Melnitschenko, P. A.: Die Rolle des Zuschlagstoffs bei der Ausbildung der Struktur des Betons (russ.). Arbeiten des Instituts für Eisenbahnverkehrswesen, Folge 86, Transport, Moskau 1966.

(5) Mtschedlow-Petrossian, O. P.: Physikalisch-chemische Grundlagen der Betontechnologie. Zeitschrift der Unionsgesellchaft für Chemie "D. I. Mendelejew".

(6) Mtschedlow-Petrosian, O. P.: Gelenkte Strukturbildung die wissenschaftliche Grundlage der Betontechnologie (russ.) Arbeiten des Stahlbeton-Forschungsinstituts. "Struktur, Festigkeit und Deformation von Betonen". Moskau, Stroiisdat 1966. 